ORIGINAL ARTICLE

\title{
Impact of antibiotics on fluorescent Pseudomonas group and Bacillus cereus group isolated from soils exposed to effluent or waste from conventional and organic pig farming
}

\author{
H.N. Chinivasagam (D, P.M. Pepper and P.J. Blackall \\ Department of Agriculture and Fisheries, Animal Research Institute, Yeerongpilly, Qld, Australia
}

Keywords

antibiotics, Bacillus, organic, piggery effluent,

Pseudomonas, soil.

\section{Correspondence \\ H. Nalini Chinivasagam, EcoSciences Precinct, Department of Agriculture and Fisheries, GPO Box 267, Brisbane, Qld 4001, Australia. E-mail: nalini.chinivasagam@daf.qld.gov.au; nalinic@me.com}

2020/1193: received 9 June 2020, revised 4 August 2020 and accepted 6 August 2020

doi:10.1111/jam.14819

\begin{abstract}
Aims: To determine if antibiotics associated with conventional pig farming have a direct role in altering the populations of key soil micro-organisms isolated from piggery environments with and without exposure to antibiotics. Methods and Results: Fluorescent Pseudomonas sp. and the Bacillus cereus group from soils adjacent to four conventional piggeries (use of antibiotics) exposed to effluent (via irrigation) and two organic piggeries (non-use of antibiotics) were assessed against nine relevant antibiotics using disc diffusion. The focus of the study was not to determine antibiotic resistance (or sensitivity) of isolates based on the manufacturer-defined sensitive break point, instead this point was used as the interpretation point to compare the populations (i.e. farm/organism combination) for the antibiotics tested. Each population was statistically analysed to determine whether the mean diameters were significantly above this selected interpretation point. Bacterial species from both environments did not show a distinct population pattern linked to the antibiotics.

Conclusions: Antibiotics associated with conventional pig farming do not have a direct role in altering the environmental populations of Pseudomonas and Bacillus sp. when assessed by population shifts.

Significance and Impact of the Study: This study confirms that an understanding of the resident soil microbiota, as compared to the transient bacteria of pig origin, is important in addressing the impact of antibiotic usage on the food-chain as a consequence of effluent re-use in, and around, pig farms.
\end{abstract}

\section{Introduction}

Piggery effluent, a potential source of antibiotic residues (Zhang et al. 2018) can be commonly used for in-shed cleaning or re-used externally, with both uses having potential impacts on human health and the natural environment (Wang et al. 2019). Antibiotic usage in food animal production varies from human usage. In food animal production, antibiotics are commonly administered therapeutically to individuals or groups as well as subtherapeutically for growth promotion (Venglovsky et al. 2009). Some classes of drugs used for animal health belong to the same classes as those used in human medicine, a further cause for concern among some quarters with respect to the development of resistance to key antibiotics (Collignon 2009).

It is well accepted that the use of antibiotics in pig production can result in the presence of antibiotic-resistant bacteria in the faeces of the treated pigs (Aarestrup et al. 1998a, 1998b). The application of waste from pig effluent ponds to land can be a source of antibiotic-resistant forms of pig-sourced gastrointestinal bacteria and of resistance genes as well as the actual antibiotics (CheeSanford et al. 2009), all of which can contribute to resistance among soil microflora. Chee-Sanford et al. (2001) have shown the potential for indigenous soil microbiota 
to mobilize tet $(\mathrm{M})$ genes in ground water associated with pig effluent lagoons.

Thus, there is the potential for antibiotic resistance genes to move from the bacteria of animal origin to indigenous soil bacteria, creating an unwanted reservoir of antibiotic resistance genes (Jensen et al. 2001). However, the presence of the pig sourced bacteria in soil environments may be transitional compared to the indigenous soil flora. Survival studies on Australian soils that received piggery effluent have shown a $90 \%$ die-off time $\left(T_{90}\right)$ of 14 and 15 days for introduced Salmonella and Escherichia coli, suggesting a low probability of long term survival of bacteria of enteric origin in soil environments (Chandler and Craven 1980). As the original source of resistance genes acquired by human pathogens originated from environmental organisms, changes in soil microbiota (as a consequence of the transfer of resistance genes) are of importance to the future of human health (Grenni et al. 2018). However, the potential impacts, arising from the usage of antibiotics within agricultural settings, to human health remain controversial. Thus, there remains a need to evaluate antibiotic resistance not just within clinical settings but also in natural environments (Schmieder and Edwards 2012).

The antibiotic residues themselves may be a source for the development of resistance among external soil organisms. Higher antibiotic concentrations than that usually present in natural ecosystems can be found in soils treated with manure (Martinez 2009), contributing to antibiotic resistance selection pressure. Such antibiotics can be bound to soil and still be active (Chander et al. 2005), although composting does degrade the antibiotics (Dolliver et al. 2008). Sengelov et al. (2003) linked tetracycline resistance levels in soil to the immediate period while the pig manure was being added, with the resistance levels returning to background after five weeks, suggesting only transient impacts in soil.

Soil micro-organisms are important inhabitants of the soil ecosystem. Soil itself is a heterogeneous habitat which harbours large genetic diversity, opening opportunities for the exchange of genetic material (Nesme and Simonet 2015). Soil is also an extensive reservoir for micro-organisms, where the top $10 \mathrm{~cm}$ of fertile soil rhizosphere can contain over two tonnes of micro-organisms (Nwosu 2001), a level that is far higher than that of the transient faecal-sourced micro-organisms. Furthermore, soil bacteria are also known to contribute to the coevolution of resistance in the presence of antibiotic biosynthesis (Laskaris et al. 2010). As an example, a total of 480 isolates of Streptomyces sp. (from urban, agricultural and forest soils) that were subjected to 21 antibiotics (natural and synthetic) at high concentrations were all multidrug resistant to an average to seven or eight of those antibiotics, highlighting the antibiotic resistance potential of soil micro-organisms (D'Costa et al. 2006). There is also evidence that residual antibiotics, resistant micro-organisms and their associated resistance genes can be transferred via waste and affect the soil community with impact on the human food chain (Thiele-Bruhn 2003). Figure 1 illustrates the two potential pathways, both directly from intensively raised pigs as well as those linked to the environment in and around intensive pig production sites that can contribute to the movement of antibiotic-resistant bacteria (or their genes). The latter indirect pathway is a situation that can occur as a consequence of waste entering the general environment.

The two key organisms used in this study were carefully selected. The genus Pseudomonas survives in a range of environments and includes species of significant environmental importance as well as species that are pathogenic to plant, animal and humans (De Souza et al. 2003). The versatile fluorescent Pseudomonas group ( $P$. aeruginosa, $P$. fluorescens and $P$. putida) has an ecological role within the soil micro-environment driven by their varied metabolic traits (Campbell et al. 1995). Similarly the genus Bacillus is central to key ecosystems (humans, water, animals and soil) with the potential to move resistance genes due to this interconnectedness across these four ecosystems (Nwosu 2001). The Bacillus cereus group (B. anthracis, B. thuringiensis, B. cereus and B. mycoides) dominates the soil environment worldwide, possess diverse physiological capabilities and are ecologically significant in soil (von Stetten et al. 1999). The transient presence of antibiotic resistance in populations of both Pseudomonas and Bacillus groups originating from soils treated with piggery manure or slurry has been previously documented (Jensen et al. 2001).

The present study explores the impact of antibiotics on normal soil flora in environments that have received piggery effluent. The study looked at environments that had received effluent from conventional piggeries that used antibiotics as well as environments that received waste from organic piggeries that did not routinely use antibiotics. Two groups of common soil organisms, the Pseudomonas fluorescens group and the B. cereus group, that had been isolated from soil exposed to either effluent or faeces for at least 10 years from six farms (four conventional and two organic) were studied and the populations compared.

\section{Methods}

\section{Piggeries}

Six south-east Queensland piggeries, specifically their soil environments, formed the basis for the study. The soil environments tested from piggeries that used antibiotics 


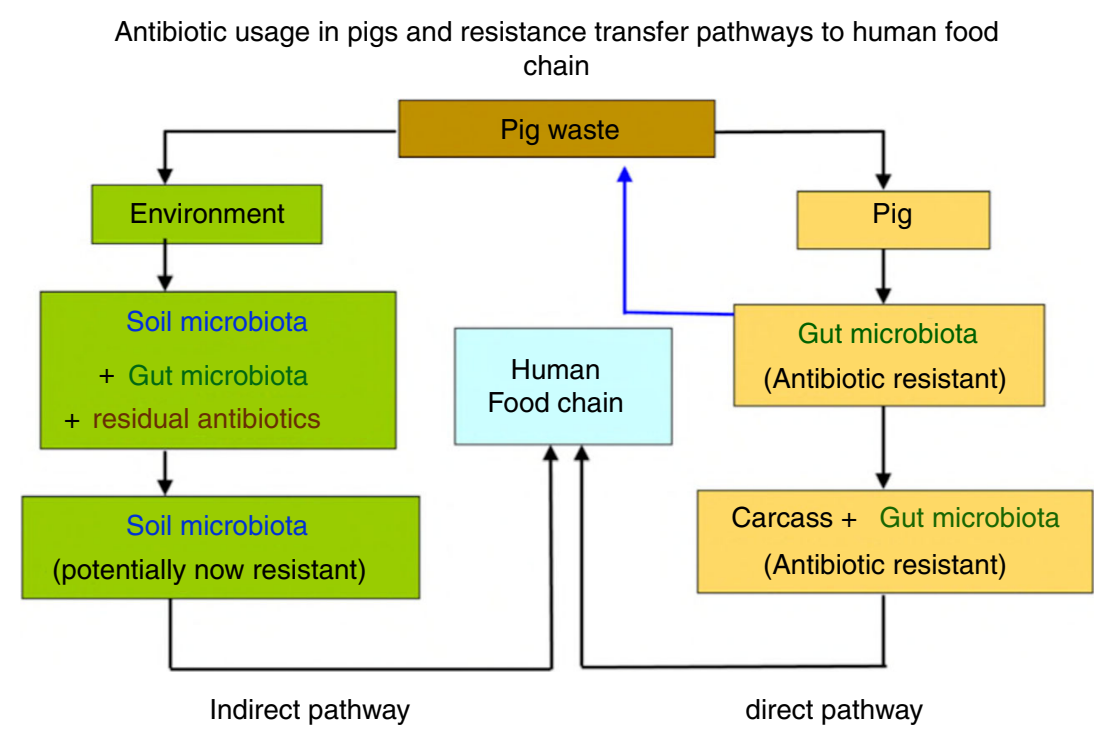

Figure 1 Potential pathways for the transmission of antibiotic-resistant organisms to the human food-chain.

were denoted as Farms A1 to A4 (where A = Antibiotics) and those from piggeries that did not use antibiotics were denoted as Farms F1 and F2 (where F = Free). The piggeries coded as A1, A2, A3 and A4 were typical continuous flow piggeries. While detailed records were not available to this study, all four piggeries had a history of the use of antibiotics, as and when necessary, for the management of herd health. Among the other piggeries, F1 was an organic free-range piggery that used antibioticfree organic pig feed and in the 10 years of operation had not used antibiotics to treat or prevent infectious diseases. The final piggery F2 had operated as an organic piggery for 10 years. Piggeries A1, A2, A3, A4 and F2 had a single use anaerobic treatment pond and an extensive (over 10 year) history of piggery effluent re-use for irrigation. The selected six piggeries were free of any particular disease problems at the time of the study. For the purpose of this study piggeries A1, A2, A3 and A4 are termed conventional piggeries and the environment receiving their effluent is termed a conventional farm environment. Relevant details of the six study piggeries are provided in Table 1.

\section{Soil samples from piggery environments}

Each of the six piggeries was visited once. Soil samples were collected from pasture (Piggeries A1, A2, A4 and F2) or crop cultivation locations (Piggery A3) that had received piggery effluent regularly for over 10 years. On Piggery F1 (free range organic), soil samples were collected from the oldest pen area which had a pig presence
Table 1 Details of the six piggeries involved in this study*

\begin{tabular}{llll}
\hline Piggery & Production System & Organic & Number of Sows \\
\hline A1 & Growers only & No & N/A \\
A2 & Farrow to Finish & No & 300 \\
A3 & Farrow to Finish & No & 300 \\
A4 & Farrow to Finish & No & 500 \\
F1 & Farrow to Finish (Free range) & Yes & 20 \\
F2 & Sows and weaners only & Yes & 500 \\
\hline
\end{tabular}

*Piggeries coded with an A have a typical antibiotic use program. Piggeries coded with a $\mathrm{F}$ have not used antibiotics for at least 10 years. $\mathrm{N} / \mathrm{A}=$ not applicable.

for over an 8 years. A 2-cm diameter sterile stainless steel soil sampler was used per sampling location to remove a soil core to a maximum depth of $4 \mathrm{~cm}$. Ten such sampling locations were selected at each farm by the random throwing of a hoop with duplicate samples collected within each hoop location and then combined. Finally, five such combined samples were composited, leading to two final samples for each farm environment. These samples, collected under aseptic conditions, were transported at room temperature and microbiological analysis was performed within $24 \mathrm{~h}$ of sample collection.

\section{Isolation and verification of fluorescent Pseudomonas sp.}

A $20 \mathrm{~g}$ aliquot of the pooled soil sample was added, under aseptic conditions, to a Whirl-pak bag. A $180 \mathrm{ml}$ volume of $0.1 \%$ peptone was added and the bag was placed on an orbital shaker for $30 \mathrm{~min}$. Tenfold dilutions 
(in $0 \cdot 1 \%$ peptone) (as $100 \mu \mathrm{l}$ aliquot) were spread on Pseudomonas agar F (Merck, Cat No. 110989, Darmstadt, Germany) and incubated at $30^{\circ} \mathrm{C}$ for $24 \mathrm{~h}$. Single colonies that showed fluorescence were picked and re-streaked onto Pseudomonas agar. The subculture plate was incubated at $30^{\circ} \mathrm{C}$ overnight. If the culture was pure and showed fluorescence, the culture was accepted as a fluorescent Pseudomonas sp. and the culture was stored frozen at $-80^{\circ} \mathrm{C}$ in $15 \%$ glycerol.

\section{Isolation and verification of Bacillus cereus group}

Isolates of the $B$. cereus group were isolated as described above, except that the 10 -fold serial dilutions were spread on B. cereus agar base (Oxoid, Cat No. CM0617) containing polymyxin B (Oxoid, Cat No. SR009E) and egg yolk (Oxoid, Cat No. SR0047, Basingstoke, UK) and incubated at $30^{\circ} \mathrm{C}$ for $24 \mathrm{~h}$. Single colonies that showed the typical colony morphology and the typical blue colouration were picked and re-streaked onto nutrient agar. The subculture plate was incubated at $30^{\circ} \mathrm{C}$ overnight. If the culture was pure and showed typical colony morphology, the culture was accepted as a member of the $B$. cereus group and the culture was stored frozen at $-80^{\circ} \mathrm{C}$ in $15 \%$ glycerol. For both organisms, all isolates were single colony picked in two serial subcultures; therefore, each isolate could be regarded as a clone.

\section{Confirmation of identification}

A random selection of 17 of the fluorescent Pseudomonas sp. isolates and 19 of the B. cereus group isolates were further identified. The fluorescent Pseudomonas sp. and the $B$. cereus group isolates were identified using the API20NE (Biomerieux, Craponne, France) and the API $50 \mathrm{CH}$ kits (Biomerieux), respectively, as recommended by the manufacturer (https://www.biomerieux.co.uk/prod uct/apir-id-strip-range).

\section{Levels of Escherichia coli}

The level of E. coli present in all soil samples was determined using the same dilutions prepared above and a three tube most probable number (MPN) method using LMX broth (Merck, Cat No. 110620) as previously described (Geissler et al. 2000).

\section{Testing of antibiotic disc diameters}

A range of antibiotics were examined using a disc diffusion methodology. Initially, the testing protocol was validated based on the National Committee for Clinical Laboratory Standards NCCLS guideline (NCCLS 2002), the guidelines recommended by the disc manufacturer. This validation work used the recommended quality control (QC) strains-Escherichia coli ATCC 25922, P. aeruginosa ATCC 27853 and Staphylococcus aureus ATCC 25922. These QC strains were then included with each test run to ensure validity. Each isolate was tested once. The antibiotics used for the two different groups of bacteria (Bacillus and Pseudomonas) are shown in Table 2. The tests were performed on commercially prepared Mueller-Hinton agar (Oxoid, Cat No. PP2096) using a Neo-SensiTabs disc (Rosco https://www.rosco.dk/?id= $9 \& c=$ Susceptibility-testing) as recommended by the manufacturer. Isolates were grown overnight on nutrient agar at $30^{\circ} \mathrm{C}$. A tryptic soy broth was inoculated and incubated at $30^{\circ} \mathrm{C}$ for around $4 \mathrm{~h}$. The isolates were then adjusted to an optical turbidity equivalent to a MacFarland 0.5 nephelometer using tryptic soy broth. The adjusted suspension was then swabbed over the surface of the required number of Mueller-Hinton plates. The antibiotic discs were applied using the specialized application device supplied by the manufacturer of the discs. The plates were then incubated at $30^{\circ} \mathrm{C}$ overnight and the zones of inhibition around the antibiotic containing discs were recorded. The zone diameters were compared with the diameter that, according to the manufacturer, equated with the sensitive interpretation point as defined by the National Committee on Clinical Laboratory Standards (NCCLS 2002). These criteria are provided in Table 2. The selected interpretation points were used for population comparison purposes (see details below) and no attempt was made to interpret the results as indicating sensitivity or resistance.

\section{Definition of populations}

For the purpose of this study, the isolates obtained from the soils exposed to piggery effluent from piggeries with antibiotic usage (A1, A2, A3 and A4) were considered as the "test population". The isolates obtained from the site exposed to manure and effluent from organically produced pigs (F1 and F2) were considered as the "control population" as a means of drawing comparisons.

\section{Statistical methods}

For each antibiotic, organism and farm combination, Gaussian curves were fitted to the various zone diameters (expressed as a percentage) using the formula

$$
y=\alpha+\frac{\beta}{\sqrt{2 \pi \sigma^{2}}} \exp \left(\frac{-(x-\mu)^{2}}{2 \sigma^{2}}\right)
$$

where $\mu=$ mean diameter; $\sigma=$ standard deviation. 
Table 2 Details of the antibiotic/bacteria combinations tested and the selected zone diameter used to compare the bacterial populations*

\begin{tabular}{llll}
\hline & \multicolumn{2}{l}{ Used with } & \\
\cline { 2 - 3 } Antibiotic & Bacillus & Pseudomonas diameter (mm) for \\
interpretation point
\end{tabular}

*The diameter selected for population comparison was the diameter suggested for the sensitive interpretation point for veterinary pathogens in a standardized methodology (NCCLS 2002). No conclusions about sensitivity or resistance were reached in this study - the interpretation point was used for population comparison purposes.

$$
\alpha+\frac{\beta}{\sqrt{2 \pi \sigma^{2}}}=\text { apex of curve. }
$$

To enable an analysis of whether the populations of the organisms in the various farm soils differed, the probability that each mean diameter was significantly $(P<0.05)$ greater than the diameter recommended by the manufacturer as the sensitive interpretation point was used. This was done to allow an interpretation point for comparison and not for the purposes of associating sensitive or resistant status to the isolates. The 95\% significance level (i.e. $P<0.05$ ) has been adopted throughout this work.

\section{Results}

\section{Bacterial levels and species diversity}

In comparison to the levels of both $P$. fluorescens and the $B$. cereus groups, the levels of $E$. coli were notably lower in both piggery environment types (i.e. $\log 1.5$ and $\log$ 1.0 MPN per gram, conventional and organic farms respectively). This was in comparison to $\log 4.7$ and $\log$ $3 \cdot 1$ CFU per gram for $P$. fluorescens and $\log 5 \cdot 3$ and $\log$ 4.0 CFU per gram for $B$. cereus groups from both conventional and organic farms respectively. The B. cereus group levels in the conventional piggery environment were significantly higher than in the organic piggery environment (Table 3 ).

The speciation of the B. cereus group and the P. fluorescens group is presented in Table 4 . While lower numbers of isolates were examined for the organic farm environment, the species diversity for the $B$. cereus group was greater for the organic farms (five species) than the conventional farms (two species). For both farm types, the dominant members of the $B$. cereus group were $B$. cereus and $B$. mycoides. For the $P$. fluorescens group, the conventional farms yielded a greater species diversity (three species) than the organic farms (one species). Ps. fluorescens was a common isolate for both farm types, although the most common species in the conventional farms ( $P$. putida) was not identified among the organic farm isolates.

\section{Population differences}

A total of 106 isolates of Pseudomonas sp. and 110 Bacillus sp. distributed across the four conventional farm environments and 60 each of both Pseudomonas sp. and Bacillus sp. across the two organic farm environments were tested. Gaussian curves were fitted to the zone diameter data for each farm/antibiotic/bacterium combination. The mean diameters were then compared to the sensitive interpretation point to allow a statistical comparison of the six populations (two organic and four conventional farm environments). In some instances, no statistical test was possible or needed. In the extreme case, for example for the ampicillin data in some farm environments, $100 \%$ of the zone diameters were $10 \mathrm{~mm}$.

\section{Pseudomonas populations}

A total of 166 Pseudomonas isolates were tested for both organic and conventional farms. Gaussian curves could not be fitted for any farm for the ampicillin data and for the following specific farm/antibiotic combinations: Farm A1/chloramphenicol, Farm A1/erythromycin, Farm A1/ kanamycin and Farm A3/tylosin populations. For the ampicillin data, three farms (A1, F1 and F2) had 100\% of

Table 3 Levels of Pseudomonas fluorescens group, Bacillus cereus group and Escherichia coli expressed as the mean log colony forming units (CFU) per gram from soil obtained from conventional and organic farm environments

\begin{tabular}{|c|c|c|c|c|c|}
\hline \multirow[b]{3}{*}{ Organisms } & \multicolumn{4}{|c|}{$\begin{array}{l}\text { Bacterial levels (log CFU per } \\
\text { gram) in soil }\end{array}$} & \multirow[b]{3}{*}{ Significance } \\
\hline & \multicolumn{2}{|c|}{ Conventional } & \multicolumn{2}{|c|}{ Organic } & \\
\hline & Mean & SE & Mean & SE & \\
\hline P. fluorescens group & 4.7 & 0.51 & $3 \cdot 1$ & 0.63 & N.S. \\
\hline B. cereus group & $5 \cdot 3$ & 0.24 & $4 \cdot 0$ & 0.34 & $P<0.05$ \\
\hline E. coli & 1.5 & 0.25 & 1.0 & 0.51 & N.S. \\
\hline
\end{tabular}


Table 4 Further identification of a selected number of isolates of Bacillus and Pseudomonas sp.

\begin{tabular}{lcc}
\hline & Number of isolates from \\
\cline { 2 - 3 } Organism & Conventional piggeries & Organic piggeries \\
\hline Bacillus cereus group & & \\
B. anthracis & 0 & 1 \\
B. cereus & 11 & 3 \\
B. firmus & 0 & 1 \\
B. megaterium & 0 & 1 \\
B. mycoides & 8 & 2 \\
Pseudomonas fluorescens group & \\
Chromobacterium & 1 & 0 \\
Burkeholderia cepacia & 0 & 1 \\
P. aeruginosa & 2 & 0 \\
P. fluorescens & 4 & 5 \\
$P$. putida & 10 & 0 \\
\hline
\end{tabular}

the isolates with the minimum zone diameters $(10 \mathrm{~mm})$ and the other three farms had 82,74 and $68 \%$ of isolates with the minimum zone diameter. Similarly, the zone diameters of Farm A1 isolates for chloramphenicol and erythromycin were clustered near the minimum as was the Farm A3 tylosin population.

For chloramphenicol, erythromycin, neomycin, tetracycline and tylosin, no population (conventional or organic) had a mean diameter significantly greater than the selected interpretation point (the tylosin curves are shown in Fig. 2). For ciprofloxacin, all mean diameters (conventional and organic) were significantly above the interpretation point (Fig. 2). For two antibiotics, there were divisions in the populations, these being kanamycin and streptomycin. For kanamycin, the Farm A2, A4 and F2 populations (two conventional and one organic) showed a mean diameter significantly greater than the interpretation point (Fig. 2). For streptomycin, only the population from Farm A3 (a conventional piggery) had a mean diameter significantly greater than the interpretation point (Fig. 2).

\section{B. cereus populations}

A Gaussian curve could not be fitted for the Farm A2/ bacitracin combination where the zone diameters were clustered near the minimum $(10 \mathrm{~mm})$. For bacitracin and tetracycline, no population (conventional or organic) had a diameter mean significantly greater than the selected interpretation point (the tetracycline curves are shown in Fig. 3). For vancomycin, all populations (conventional and organic) had diameter means significantly greater than the interpretation point (Fig. 3). For the rest of the farm/antibiotic combinations there was no trend based on antibiotic practices. As an example, the A1, A2 and A4 (conventional) and F1, F2 (organic) population mean diameters were above the interpretation point for erythromycin and gentamicin but not the A3 (conventional) population. Interestingly, F1 (an organic population), had the highest number of antibiotics (ciprofloxacin, erythromycin, gentamicin, streptomycin and tylosin) with zone diameters above the interpretation point. The full details are shown in Table 5 and the curves for erythromycin, streptomycin, vancomycin and tetracycline are shown in Fig. 3.

\section{Discussion}

Concerns surrounding the use of antibiotics both in human medicine as well as animals have attracted considerable attention in terms of contributing to the dissemination of resistance genes. It is estimated that up to $75 \%$ of the antibiotics used in animal production are not absorbed by the animal and end up in the waste possibly entering the effluent pond, along with excreted antibiotic-resistant forms of gastrointestinal bacteria (CheeSanford et al. 2009). In conventional Australian piggeries, pigs are commonly raised in sheds with a combination of solid and slatted floors and the collected effluent is transferred to a ponding system to enable treatment following a resident time. In Australia, effluent is commonly disposed to agricultural land (Payne 1990), with the potential for transfer of resistance genes or resistant organisms.

Antibiotics in therapy and growth promotion can have a role in the transmission of antibiotic-resistant bacteria and resistance genes in ecosystems such as the soil and aquatic environments (Witte 2000). These antibiotics can have an impact on the indigenous bacterial populations which are better adapted to survive and persist in natural soil ecosystems (Chee-Sanford et al. 2009). Antibiotic-resistant organisms such as E. coli and enterococci have been isolated from Australian pigs and the piggery environments (Hart et al. 2004), possibly as a consequence of antibiotic usage. However, tetracycline resistance levels in soil microbiota that received pig manure slurry have been shown to be only temporarily influenced, with those resistance levels returning to the control soil levels with time (Sengelov et al. 2003). This indicates the possibility of the return to normal situations when antibiotic levels decline. A similar result, a return to prior levels of tylosin resistance at 90 days after a one-off addition of tylosin to soil supporting the growth of alfalfa, was found by Demoling and Baath (2008).

The current study compared soil bacteria from four sites with a long history of exposure to piggery effluent from conventional piggeries (that have used antibiotics) with two sites that have had either a history of exposure 

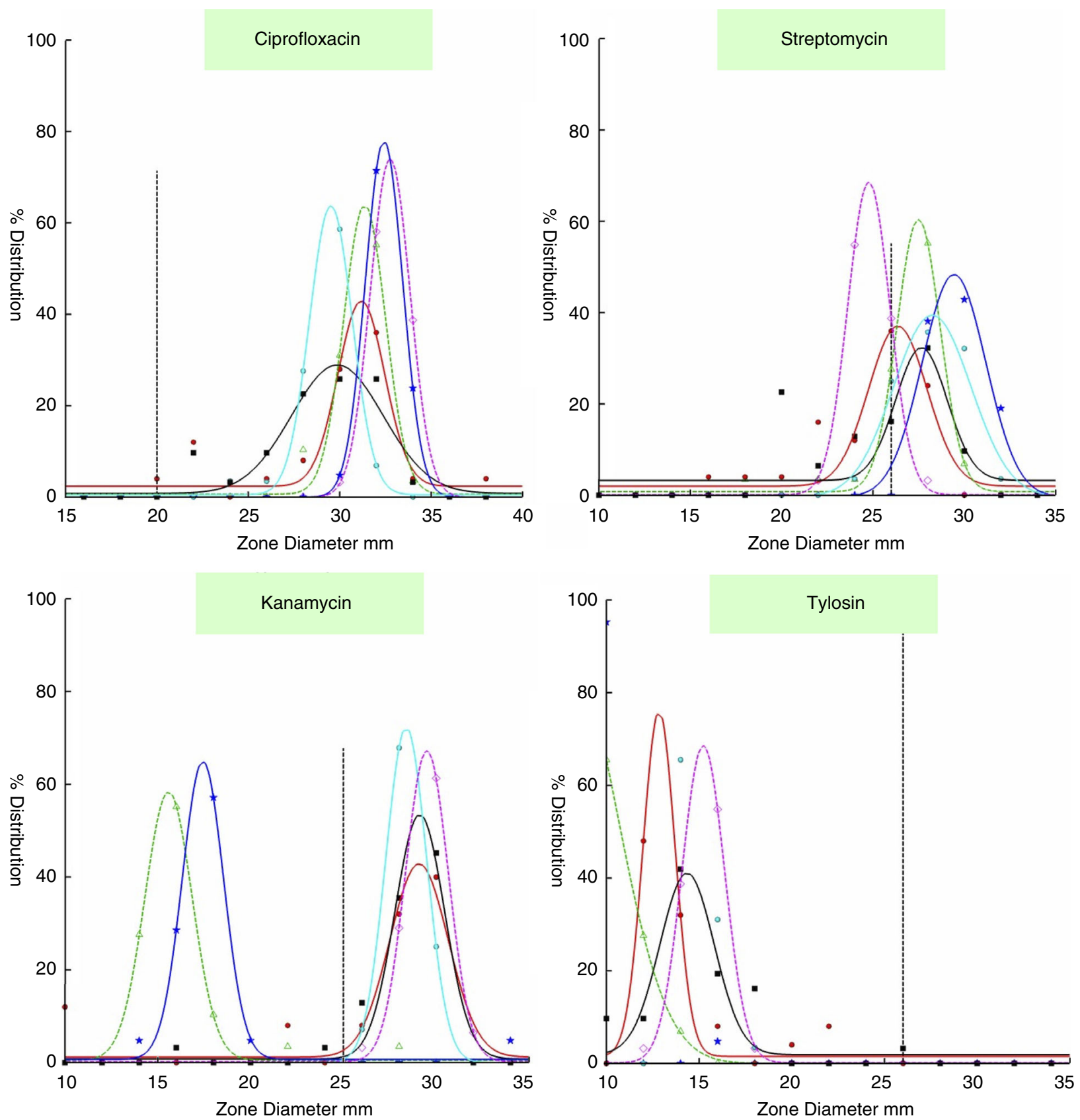

Figure 2 Population distribution for the Pseudomonas fluorescens group from both conventional (A1 @ , A2 $\mathbf{\square}, \mathrm{A} 3 \star$, and A4 e) and organic (F1 $\triangle$ and F2 $\diamond$ ) farm environments (i.e. test and control populations respectively). The vertical dotted line represents the interpretation point for comparing the various bacterial populations.

to manure from pigs produced by a certified free range organic piggery or exposure to effluent from a certified organic piggery. These latter two sites had no exposure to antibiotics and thus represented the indigenous soil populations associated with piggery environments exposed to normal pig waste (effluent or faeces). Some of the antibiotics included in this study were selected as they are approved for either therapeutic or growth promotion use in pig production in Australia-ampicillin, erythromycin, neomycin, tetracycline and tylosin. Other agents were selected because they represented classes of antibiotic approved for use; phenicols (chloramphenicol) and aminoglycosides (gentamicin, kanamycin and streptomycin), although these agents themselves are not 

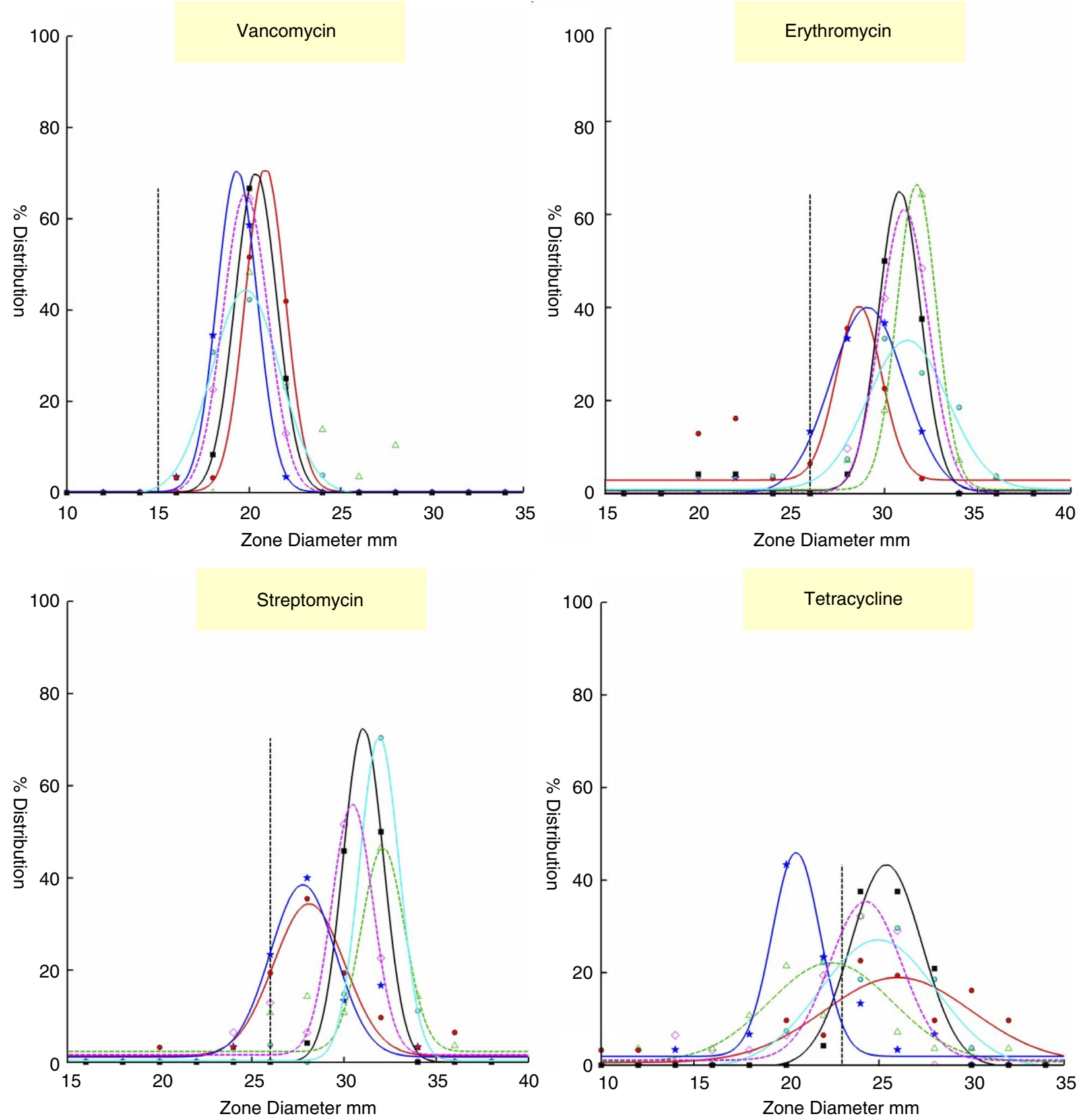

Figure 3 Population distribution for the Bacillus cereus group from both conventional (A1 $0, A 2 \mathbf{\square}, A 3 \star$ and $A 4 \bigcirc$ ) and organic (F1 $\triangle$ and F2 $\checkmark)$ farm environments (i.e. test and control populations respectively). The vertical dotted line represents the interpretation point for comparing the various bacterial populations.

registered. The final set of antibiotics represented classes that are not approved for use in pigs in Australia, polypeptides (bacitracin), fluoroquinolones (ciprofloxacin) and glycopeptides (vancomycin) with the latter two being included because of their importance in human medicine. The natural soil environment also contains several antibiotic-producing micro-organisms potentially having a unique ecological role in natural, nonclinical, environments to inhibit competitors (Fajardo and Martínez 2008). It is thus possible that the presence of antibiotic resistance genes in indigenous soil bacteria may not have a direct link with transferred animal waste. The 
Table 5 Summary of statistically significant probability differences in the various Bacillus populations

\begin{tabular}{ll}
\hline Antibiotic & $\begin{array}{l}\text { Populations with a mean zone diameter signifi- } \\
\text { cantly }(P<0.05) \text { above the interpretation point }\end{array}$ \\
\hline $\begin{array}{l}\text { Chloramphenicol } \\
\text { Ciprofloxacin }\end{array}$ & $\begin{array}{c}\text { Farm A2 (conventional) } \\
\text { Farms A1 and A4 (conventional) and Farms F1 } \\
\text { and F2 (organic) }\end{array}$ \\
Erythromycin & $\begin{array}{l}\text { Farm A1, A2 and A4 (conventional) and Farms F1 } \\
\text { and F2 (organic) }\end{array}$ \\
Gentamycin & $\begin{array}{c}\text { Farm A1, A2 and A4 (conventional) and Farm F1 } \\
\text { and F2 (organic) }\end{array}$ \\
Streptomycin & $\begin{array}{c}\text { Farms A2 and A4 (conventional) and Farms F1 } \\
\text { and F2 (organic) }\end{array}$ \\
Tylosin & Farms A2 and A4 (conventional) and Farm F1 \\
& (organic)
\end{tabular}

For bacitracin and tetracycline, no population (conventional or organic) had a mean zone diameter significantly above the interpretation point. For vancomycin, all the populations had a mean zone diameter significantly above the interpretation point.

current study was designed to provide further understanding to address this issue.

The organisms selected to represent the indigenous microbial populations, that is the fluorescent Pseudomonas sp. and the B. cereus group were by far dominant in soil (from $3 \cdot 1$ to $5.3 \log$ CFU per gram) compared to the indicator organism E. coli (1.0-1.5 log CFU per gram) regardless of the farming type (conventional or organic) though $E$. coli is typically present at high levels $\left(10^{5} \mathrm{CFU}\right.$ per $\left.100 \mathrm{ml}\right)$ in piggery effluent (Chinivasagam et al. 2004). The key outcome of the study was that bacterial populations (336 isolates of Pseudomonas and Bacillus) sourced from the two major environments that is exposed to normal piggery (216 isolates) and organic piggery waste (120 isolates) did not show a distinct statistically significant population pattern distribution that could be associated with the influence of antibiotics, based on the selected interpretation point. Any impact on the populations as a result of antibiotic pollution in the environment can be interpreted as being above the normal (Martinez 2009). The normal in the present study being the population curves of the $P$. fluorescens group (60 isolates) and the $B$. cereus group (60 isolates) from the organic piggeries. These population curves are representative of the indigenous flora in the piggery environment. If antibiotics in pig effluent were changing the soil flora, then the populations should consistently show a significant difference between the conventional farms and the organic farms. This was not the case in the present study, suggesting no influence of the presence of antibiotics.

Both Pseudomonas sp. (in particular P. fluorescens) and Bacillus species (in particular B. subtilis) are common soil bacteria (van Elsas et al. 1986) and thus are part of the typical soil flora in any environment such as those around piggeries. A Danish study (Jensen et al. 2001) used both the $P$. fluorescens and the B. cereus groups from pig farm soil to study the antimicrobial susceptibility of normal soil microbiota exposed to piggery waste and reported a temporary rise in resistance levels, compared to soil environments that had not received piggery waste. While the focus of the current study was not the same as in the Jensen et al. (2001) study, the choice of flora was retained. Importantly, a variation of Jensen et al. (2001) was adopted-an examination of the population shifts of both Pseudomonas and Bacillus groups in farm soils with exposure to antibiotics in pig waste as compared to those in soils exposed to organic pig waste that did not contain antibiotics.

Both Pseudomonas and Bacillus genera contain considerable species diversity and this diversity was reflected among the representative isolates identified in the present study. Among the five taxa identified in the P. fluorescens group in the current study, three species ( $P$. aeruginosa, $P$. fluorescens and P. putida) are common in both industrial as well as agricultural soils (Campbell et al. 1995). Similarly, representatives of the $B$. cereus group ( $B$. anthracis, B. cereus and B. mycoides), were also isolated during the present study, confirming species diversity among the tested isolates from the varied piggery environments.

For the Pseudomonas populations, there were two antibiotics where at least one population significantly differed from the remaining populations. For one of these antibiotics, kanamycin, one conventional farm population and one organic farm population showed a significant difference to the remaining populations. This finding indicates that the shift in population is not consistently associated with use of antibiotics. The other antibiotic was streptomycin, with the mean diameter from one farm that used antibiotics (Farm A3), being significantly different from the interpretation point whereas the other means from three conventional and two organic farms were not. Importantly, streptomycin has been banned from use in food producing animals in Australia since 1998 (Anon 1999). This lack of a consistent difference between the organic and conventional piggeries combined with the lack of use of the antibiotic since 1998 suggests that the population difference detected for streptomycin has no connection with the use of antibiotics on the farm concerned (Farm A3). Overall, there was no marked trend that would indicate that conventional piggery effluent that could contain antibiotics was associated with shifts in bacterial populations away from the interpretation point compared with populations associated with organic pig waste/effluent. 
For the Bacillus populations, there were six antibiotics where at least one population significantly differed from the remaining populations (across conventional and organic). For three of these antibiotics (chloramphenicol, ciprofloxacin and gentamicin), there has never been any formal approval for their use in Australian pigs while for one antibiotic (streptomycin, significantly different for one conventional farm), formal approval has been withdrawn for a number of years (Anon 1999). Hence, as these four antibiotics were either never used or not used for a number of years, there seems little likelihood that the population differences seen in this study are associated with direct exposure to these antibiotics. There were significant population differences detected for two antibiotics that are approved for use in Australian pigs (erythromycin and tylosin). For erythromycin, there was one population (Farm A3-a conventional piggery) where this population was not significantly likely to have a mean zone diameter above the interpretation point. For the other antibiotic (tylosin), two of the four conventional (Farms A2 and A4) and one of the two organic (Farm F1) piggeries had populations with a statistically significant probability of mean zone diameters above the interpretation point. As remaining populations were both organic (Farm F2) and conventional (Farms A1 and A3), there is no clear conclusion that these divisions in populations were due to exposure of the soil flora to effluent containing tylosin.

The population differences seen in the current study must have a genetic basis that is the presence of genes driving antibiotic resistance. Importantly, antibiotic resistance genes have been located in pristine environments not likely to be contaminated by humans-suggesting that the primary function of these antibiotic resistance genes may not necessarily be antibiotic resistance (Martinez 2009). Indeed, it has been suggested that genes, which contribute to antibiotic resistance in pathogenic bacteria, may serve a very different function for the relevant environmental host, in environments such as soil (Nesme and Simonet 2015). Moreover, soil bacteria demonstrate coevolution of resistance in the presence of antibiotic biosynthesis as a means of adopting to antibiosis during competition for resources in soil environments (Laskaris et al. 2010). Such phenomena can explain the demonstration of resistance in bacteria from environments free of antibiotic usage. There are also a number of studies that show a sharing of structural and functional characteristics in both heavy metal and antibiotic resistance systems as reviewed by Baker-Austin et al. (2006). As an example, it is known that copper resistance is linked with macrolide (tylosin resistance) (Hasman and Aarestrup 2002). Copper levels in piggery waste have been associated with copper in pig feed (Robinson et al.
1971). The presence of tetracycline resistance genes in the soil around pig feedlots in China is suggested to have occurred via lagoon seepage or manure to the soil $(\mathrm{Wu}$ et al. 2010). A key issue with the Wu et al. (2010) study is that the source of the genes could be not be definitely attributed to either the transient swine faecal population (e.g. faecal E. coli) following effluent application to the soil or the natural soil microbiota. Thus, it is possible that interactions between soil flora and heavy metals (both those naturally present and added via feed waste) as well as other stress factors may explain the differing population distribution patterns observed in the present study, particularly those associated with antibiotics that have not been used in Australian pigs.

Overall this study has found little evidence that the presence of antibiotics linked to conventional pig farming have a direct role in altering the populations of soil microbiota in terms of zone diameter measurements in a disc diffusion methodology. This further reiterates the fact that the issue of antibiotic resistance in environments such as soil (that have no direct link to the production environment) could be influenced by a range of complex natural interactions. The overall study provides outcomes that should enable the development of guidelines to address some of the concerns surrounding the re-use of piggery effluent for agricultural purposes.

\section{Acknowledgements}

This work was funded by Australian Pork Limited. The co-operation and support of the management of the six piggeries involved in this study is gratefully acknowledged. The provision of antimicrobial discs by Rosco, Australia is also gratefully acknowledged.

\section{Conflict of Interests}

There are no conflicts of interests arising from this study.

\section{References}

Aarestrup, F.M., Bager, F., Jensen, N.E., Madsen, M., Meyling, A. and Wegener, H.C. (1998a) Resistance to antimicrobial agents used for animal therapy in pathogenic-, zoonoticand indicator bacteria isolated from different food animals in Denmark: a baseline study for the Danish Integrated Antimicrobial Resistance Monitoring Programme (DANMAP). Acta Path Microbiol Immunol Scand 106, 745-770.

Aarestrup, F.M., Bager, F., Jensen, N.E., Madsen, M., Meyling, A. and Wegener, H.C. (1998b) Surveillance of antimicrobial resistance in bacteria isolated from food animals to antimicrobial growth promoters and related 
therapeutic agents in Denmark. Acta Path Microbiol

Immunol Scand 106, 606-622.

Anon (1999) Injectable streptomycin banned in food animals. Aust Vet J 77, 486.

Baker-Austin, C., Wright, M.S., Stepanauskas, R. and McArthur, J.V. (2006) Co-selection of antibiotic and metal resistance. Trends Microbiol 14, 176-182.

Campbell, J.I.A., Jacobsen, C.S. and Sorensen, J. (1995) Species variation and plasmid incidence among fluorescent Pseudomonas strains isolated from agricultural and industrial soils. FEMS Microbiol Ecol 18, 51-62.

Chander, Y., Kumar, K., Goyal, S.M. and Gupta, S.C. (2005) Antibacterial activity of soil-bound antibiotics. J Environ Qual 234, 1952-1957.

Chandler, D.S. and Craven, J.A. (1980) Relationship of soil moisture to survival of E. coli and S. typhimurium in soils. Aus J Agric Res 31, 547-555.

Chee-Sanford, J.C., Aminov, R.I., Krapac, I.J., GarriguesJeanjean, N. and Mackie, R.I. (2001) Occurrence and diversity of tetracycline resistance genes in lagoons and groundwater underlying two swine production facilities. Appl Environ Microbiol 67, 1494-1502.

Chee-Sanford, J.C., Mackie, R.I., Koike, S., Krapac, I.G., Lin, Y.-F., Yannarell, A.C., Maxwell, S. and Aminov, R.I. (2009) Fate and transport of antibiotic residues and antibiotic resistance genes following land application of manure waste. J Environ Qual 38, 1086-1108.

Chinivasagam, H.N., Thomas, R.J., Casey, K., McGahan, E., Gardner, E.A., Rafiee, M. and Blackall, P.J. (2004) Microbiological status of piggery effluent from 13 piggeries in the south east Queensland region of Australia. J Appl Microbiol 97, 883-891.

Collignon, P. (2009) Resistant Escherichia coli - we are what we eat. Clin Inf Dis 49, 202-204.

D'Costa, V.M., McGrann, K.M., Hughes, D.W. and Wright, G.D. (2006) Sampling the antibiotic resistome. Science 311, 374-377.

Demoling, L.A. and Baath, E. (2008) No long-term persistence of bacterial pollution-induced community tolerance in tylosin-polluted soil. Environ Sci Technol 42, 6917-6921.

De Souza, J.T., Mazzola, M. and Raaijmakers, J.M. (2003) Conservation of the response regulator gene gacA in Pseudomonas species. Environ Microbiol 5, 1328-1340.

Dolliver, H., Gupta, S. and Noll, S. (2008) Antibiotic degradation during manure composting. J Environ Qual 37, 1245-1253.

Fajardo, A. and Martínez, J.L. (2008) Antibiotics as signals that trigger specific bacterial responses. Curr Opinion Microbiol 11, 161-167.

Geissler, K., Manafi, M., Amoros, I. and Alonso, J.L. (2000) Quantitative determination of total coliforms and Escherichia coli in marine waters with chromogenic and fluorogenic media. J Appl Microbiol 88, 280-285.
Grenni, P., Ancona, V. and Caracciolo, A.B. (2018) Ecological effects of antibiotics on natural ecosystems: a review. Microchem J 136, 25-39.

Hart, W.S., Heuzenroeder, M.W. and Barton, M.D. (2004) Antimicrobial resistance in Campylobacter spp., Escherichia coli and enterococci associated with pigs in Australia. $J$ Vet Med Series B 51, 216-221.

Hasman, H. and Aarestrup, F.M. (2002) tcrB, a gene conferring transferable copper resistance in Enterococcus faecium: Occurrence, transferability, and linkage to macrolide and glycopeptide resistance. Antimicrob Res Chemother 46, 1410-1416.

Jensen, L.B., Baloda, S., Boye, M. and Aarestrup, F.M. (2001) Antimicrobial resistance among Pseudomonas spp. and the Bacillus cereus group isolated from Danish agricultural soil. Environ Int 26, 581-587.

Laskaris, P., Tolba, S., Calvo-Bado, L. and Wellington, L. (2010) Coevolution of antibiotic production and counterresistance in soil bacteria. Environ Microbiol 12, 783-796.

Martinez, J.L. (2009) Environmental pollution by antibiotics and by antibiotic resistance determinants. Environ Pollut 157, 2893-2902.

National Committee for Clincal Laboratory Standards (NCCLS) (2002) Performance Standards for Antimicrobial Disk and Dilution Susceptability Tests for Bacteria Isolated from Animals; Approved Standard Second Edition. NCCLS document. M31-A2. (ISBN 1 - 56238-461-9). Wayne, PA: NCCLS. 19087 - 1898.

Nesme, J. and Simonet, P. (2015) The soil resistome: a critical review on antibiotic resistance origins, ecology and dissemination potential in telluric bacteria. Environ Microbiol 17, 913-930.

Nwosu, V.C. (2001) Antibiotic resistance with particular reference to soil microorganisms. Res Microbiol 152, 421-430.

Payne, R.W. (1990) Effluent disposal. In Pig Production in Australian ed. Gardner, J.A., Dunkin, A.C. and Lloyd, L.C. Sydney: Butterworths.

Robinson, K., Draper, S.R. and Gelman, A.L. (1971) Biodegradation of pig waste: Breakdown of soluble nitrogen compounds and the effect of copper. Environ Pollut 2, 49-56.

Schmieder, R. and Edwards, R. (2012) Insights into antibiotic resistance through metagenomic approaches. Future Microbiol 7, 73-89.

Sengelov, G., Agerso, Y., Halling-Sorensen, B., Baloda, S.B., Andersen, J.S. and Jensen, L.B. (2003) Bacterial antibiotic resistance levels in Danish farmland as a result of treatment with pig manure slurry. Environ Int 28, 587-595.

Thiele-Bruhn, S. (2003) Pharmaceutical antibiotic compounds in soils - a review. J. Plant Nutr Soil Sci 166, 145-167.

van Elsas, J.D., Dijkstra, A.F., Govaert, J.M. and van Veen, J.A. (1986) Survival of Pseudomonas fluorescens and Bacillus subtilis introduced into two soils of different 
texture in field microplots. FEMS Microbiol Lett 38, 151-160.

Venglovsky, J., Sasakova, N. and Placha, I. (2009) Pathogens and antibiotic residues in animal manures and hygienic and ecological risks related to subsequent land application. Biores Technol 100, 5386-5391.

von Stetten, F., Mayr, R. and Scherer, S. (1999) Climatic influence on mesophilic Bacillus cereus and psychrotolerant Bacillus weihenstephanensis populations in tropical, temperate and alpine soil. Environ Microbiol 1, 503-515.

Wang, S., Ma, X., Wang, Y., Du, G., Tay, J.H. and Li, J. (2019) Piggery wastewater treatment by aerobic granular sludge: granulation process and antibiotics and antibiotic-resistant bacteria removal and transport. Biores Technol 273, 350-357.

Witte, W. (2000) Ecological impact of antibiotic use in animals on different complex microflora: environment. Int J Ant Ag 14, 321-325.

Wu, N., Qiao, M., Zhang, B., Cheng, W.-D. and Zhu, Y.-G. (2010) Abundance and diversity of tetracycline resistance genes in soils adjacent to representative swine feedlots in China. Environ Sci Technol 44, 6933-6939.

Zhang, M., Liu, Y.-S., Zhao, J.-L., Liu, W.-R., He, L.-Y., Zhang, J.-N., Chen, J., He, L.-K. et al. (2018) Occurrence, fate and mass loadings of antibiotics in two swine wastewater treatment systems. Sci Total Environ 639, 1421-1431. 